\section{Commentary: Is there life after cardiac death? Considering the challenges of heart donation after circulatory death}

\author{
Alexander Raskin, MD, ${ }^{\mathrm{a}}$ Farhan Zafar, MD, ${ }^{\mathrm{b}}$ and \\ David L. S. Morales, MD $^{\mathrm{b}}$
}

Rajab and colleagues ${ }^{1}$ provide an excellent synopsis on the current state of cardiac donation after circulatory death (DCD). The authors highlight a key issue of more patients being listed than are being transplanted each year. ${ }^{1}$ As a result, managing and minimizing waitlist mortality has become paramount. Increased use of DCD has been proposed as a means to increase the donor pool by as much as $15 \%$ to $30 \%,{ }^{2}$ but this may be an overestimation. In the modern era, DCD continues to be minimally employed, with just more than 100 adult cases reported, almost all outside the United States. ${ }^{3}$ Several barriers may stand in the way of wider adoption of DCD, which is mired with legal and ethical considerations. The legal pronouncement of cardiac death revolves around the irreversible cessation of cardiopulmonary function. However, the required resuscitation and restoration of cardiac activity needed to carry out DCD transplantation may violate the tenant of irreversibility. Another consideration is that the legal definition of death may vary between jurisdictions, ${ }^{4}$ and this can make it harder to standardize widely adaptable DCD protocols. For example, some approaches call for ante-mortem placement of extracorporeal membrane oxygenation cannulas, which has been deemed unacceptable in certain institutions and jurisdictions. Variability in mandated standoff time, the time between circulatory arrest, and declaration of death may further alter warm ischemic time and graft quality. These

From the Divisions of a Pediatric Cardiology, and ${ }^{\mathrm{b}}$ Pediatric Cardiothoracic Surgery, Cincinnati Children's Hospital Medical Center, Cincinnati, Ohio.

Disclosures: Authors have nothing to disclose with regard to commercial support.

Received for publication March 25, 2020; revisions received March 25, 2020; accepted for publication March 27, 2020; available ahead of print April 11, 2020. Address for reprints: David L. S. Morales, MD, The Heart Institute, Cincinnati Children's Hospital Medical Center, 3333 Burnet Ave, MLC 2003, Cincinnati, OH 45229-3026 (E-mail: David.Morales@cchmc.org).

J Thorac Cardiovasc Surg 2021;161:1344-5

0022-5223/ $\$ 36.00$

Copyright $₫ 2020$ Published by Elsevier Inc. on behalf of The American Association for Thoracic Surgery

https://doi.org/10.1016/j.jtcvs.2020.03.088

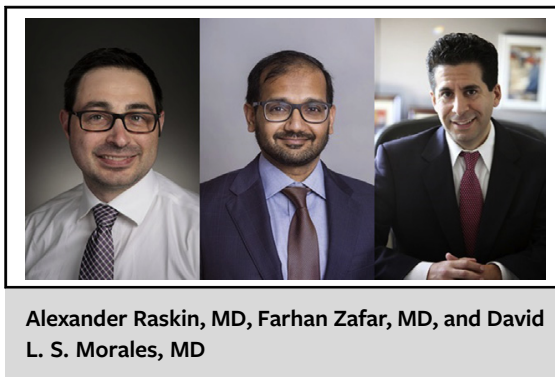

CENTRAL MESSAGE

Cardiac donation after circulatory death may not be the solution to the currently evergrowing waitlist.

considerations are only amplified when trying to employ the DCD strategy internationally.

Data from adult DCD recipients have demonstrated greater rates of immediate graft dysfunction and increased extracorporeal membrane oxygenation use. Intermediate survival seems to be equivocal for adult DCD recipients, but the overall reported numbers are limited. ${ }^{5}$ Pediatric DCD outcomes, based on 21 reported cases to the International Society for Heart and Lung Transplantation Registry, are less encouraging. According to that data, DCD recipients had increased graft dysfunction and decreased 1 -year survival of $61 \%$ versus $91 \%$ compared with donation after brain death. ${ }^{6}$ Recipient selection factors could have contributed to the survival outcomes. Unfortunately, donor characteristics were not reported in that series. These results may explain why DCD accounted for $0.5 \%$ of pediatric and less than $1 \%$ of adult transplants over the last 2 decades. ${ }^{6,7}$

Perhaps we should maximize our current pool of donors, since up to $44 \%$ of pediatric and $48 \%$ of adult available allografts are discarded. Registry data from the Organ Procurement and Transplantation Network demonstrate that donor use has declined with time. Despite seemingly more stringent donor selection, recipient survival has not improved significantly. 8,9 Many of the discarded hearts would have likely resulted in favorable clinical outcomes. In fact, adults who received adolescent allografts that were initially rejected by all pediatric centers went on to have excellent results. ${ }^{10}$ At this time, it is unclear how many of the unselected allografts would be appropriate for donation, but clearly some would be. The provincial protocols and beliefs about accepting organs that vary at each program have led to our inability to maximize the donor 
pool. More work using machine learning needs to be done to better understand and standardize the donor selection process while minimizing existing variability. We should be able to improve donor selection and use without adversely impacting recipient survival, in fact, improving it for the community as a whole. Perhaps there is life after cardiac death via donation, but we do not believe it will flourish in its current form or in the present system, as the past decade has revealed. Perhaps, the use of technology like the TransMedics Organ Care System (TransMedics, Andover, Mass) to resuscitate and evaluate the organs after cardiac death will bring life to DCD. Results from the EXPAND trial will be informative in this regard.

\section{References}

1. Rajab TK, Jaggers J, Campbell D. Heart transplantation following donation after cardiac death: history, current techniques, and future. J Thorac Cardiovasc Surg. 2021;161:1335-40.

2. White CW, Messer SJ, Large SR, Conway J, Kim DH, Kutsogiannis DJ, et al. Transplantation of hearts donated after circulatory death. Front Cardiovasc Med. 2018;5:8.
3. Macdonald P, Dhital K. Heart transplantation from donation-after-circulatorydeath (DCD) donors: back to the future - evolving trends in heart transplantation from DCD donors. J Heart Lung Transplant. 2019;38:599-600.

4. Tchana-Sato V, Ledoux D, Detry O, Hans G, Ancion A, D'Orio V, et al. Successful clinical transplantation of hearts donated after circulatory death using normothermic regional perfusion. J Heart Lung Transplant. 2019;38:593-8.

5. Chew HC, Iyer A, Connellan M, Scheuer S, Villanueva J, Gao L, et al. Outcomes of donation after circulatory death heart transplantation in Australia. J Am Coll Cardiol. 2019;73:1447-59.

6. Kleinmahon JA, Patel SS, Auerbach SR, Rossano J, Everitt MD. Hearts transplanted after circulatory death in children: analysis of the Internationa Society for Heart and Lung Transplantation Registry. Pediatr Transplant. 2017; 21:e13064.

7. Khush KK, Cherikh WS, Chambers DC, Harhay MO, Hayes D Jr, Hsich E, et al The international thoracic organ transplant registry of the International Society for Heart and Lung Transplantation: thirty-sixth adult heart transplantation report - 2019; focus theme: donor and recipient size match. J Heart Lung Transplant. 2019;38:1056-66.

8. Riggs KW, Kroslowitz BJ, Chin C, Zafar F, Morales DLS. Pondering higher-risk pediatric heart donors: can we use more? Ann Thorac Surg. November 14, 2019 [Epub ahead of print].

9. Khush KK, Zaroff JG, Nguyen J, Menza R, Goldstein BA. National decline in donor heart utilization with regional variability: 1995-2010. Am J Transplant. 2015;15:642-9.

10. Zafar F, Rizwan R, Lorts A, Bryant R III, Tweddell JS, Chin C, et al. Implications and outcomes of cardiac grafts refused by pediatric centers but transplanted by adult centers. J Thorac Cardiovasc Surg. 2017;154:528-36.e1. 